\title{
Provider payments and patient charges as policy tools for cost-containment: How successful are they in high-income countries?

\author{
Guy Carrin*1 and Piya Hanvoravongchai ${ }^{1,2}$
}

Address: ${ }^{1}$ Department of Health Financing and Stewardship, World Health Organization, Geneva, Switzerland and ${ }^{2}$ Current address: Health Systems Research Institute in Bangkok, Thailand

Email: Guy Carrin* - carring@who.int; Piya Hanvoravongchai - piya@hsrint.hsri.or.thi

* Corresponding author

Published: 3I July 2003

Human Resources for Health 2003, I:6

This article is available from: http://www.human-resources-health.com/content/I/I/6

(c) 2003 Carrin and Hanvoravongchai; licensee BioMed Central Ltd. This is an Open Access article: verbatim copying and redistribution of this article are permitted in all media for any purpose, provided this notice is preserved along with the article's original URL.
Received: 14 May 2003

Accepted: 31 July 2003

\begin{abstract}
In this paper, we focus on those policy instruments with monetary incentives that are used to contain public health expenditure in high-income countries. First, a schematic view of the main cost-containment methods and the variables in the health system they intend to influence is presented. Two types of instruments to control the level and growth of public health expenditure are considered: (i) provider payment methods that influence the price and quantity of health care, and (ii) cost-containment measures that influence the behaviour of patients. Belonging to the first type of instruments, we have: fee-for-service, per diem payment, case payment, capitation, salaries and budgets. The second type of instruments consists of patient charges and reference price systems for pharmaceuticals. Secondly, we provide an overview of experience in high-income countries that use or have used these particular instruments. Finally, the paper assesses the overall potential of these instruments in cost-containment policies.
\end{abstract}

\section{Introduction}

While many low-income developing countries still need to muster an appropriate and sometimes even a minimum amount of resources, many high-income countries are addressing the question of how to contain their health care costs. This question is not brand new, however. OECD countries already became confronted with this question some 30 to 40 years ago, when health expenditure grew almost twice as fast as Gross Domestic Product (GDP). A downward economic cycle in the 1970s as well as increased ageing of the population enhanced the concern to control health care costs[1].

Cost-containment as a policy issue is related to the question what the right amount is that countries should spend on health care. Society's preferences, and not just econom- ics, have an important impact on choosing the appropriate amount of care. For example, an increase in the share of health expenditure in GDP is not just to be understood as a cost explosion, but could simply be a response to population's preferences for more and better care. This illustrates that it will be tedious to establish when costcontainment is exactly needed.

An additional question is whether cost-containment should be directed at total expenditure or public expenditure on health. We chose to focus on public expenditure on health, in view of an overriding and steady concern about cost-containment by governmental and quasi-governmental institutions. As to the need for cost-containment, few countries use precise criteria that would trigger cost-containment measures. It seems to be accepted 
though that the aim of cost-containment is to either stabilize public health expenditure per capita or to moderate the growth rate of public expenditure for health per capita. It is often taken as evidence of moderation when the latter growth rate is less than the growth rate of GDP per capita.

Policy makers have resorted to a variety of cost-containment instruments. First, there are measures impacting directly upon the supply of health services or the inputs necessary to ensure this supply. Examples are measures to control new infrastructure, medical technology and quantities of medical personnel, and to influence the quantity and structure of health services through treatment protocols and prescribing guidelines and subsequent monitoring of clinical behaviour. Another example is influencing the supply of ambulatory vs. inpatient care as a result of a gatekeeper's role for GPs. There are also monetary incentives operating via provider payment mechanisms and that influence the supply of health services. Examples are budgets for hospitals and GPs, and other negotiated ways to pay providers, such as fee-for-services. Secondly, there are measures on the demand side of the health sector, attempting to influence patients' behaviour via non-monetary (e.g. drug information, information on prevention etc.) and monetary incentives such as patient charges or user fees.

We recognize that the above-mentioned instruments do not have cost-containment as a unique objective. The supply-side measures may also have an impact on health services productivity, quality of care, the distribution of resources, and on shortages and surpluses of human resources for health. And some of the demand-side measures may impact upon individuals' access to health care. However, in this paper, we focus on the cost-containment potential of these policy tools. In addition, only those policy tools will be analyzed that incorporate monetary incentives. Policy tools without monetary incentives and that belong to the areas of information, education and regulation will therefore not be dealt with in this particular paper. We also summarize the experience in high-income countries; all of the countries referred to belong to the OECD or the European Union. In the next section, a schematic view is presented of the cost-containment methods that will be discussed. In section 2, we analyze the experience with these policy tools and their effects on cost-containment. The levels and trends in administrative costs will be addressed in section 3 . Finally in section 4 , we provide a summary table that compares the expected effectiveness of the various tools in terms of cost-containment.

\section{Cost-containment methods: a schematic view}

In Figure 1, we present a schematic view of the main costcontainment methods and the variables in the health system they intend to influence. For expository's sake, we assume that health care is provided in two sectors of the health system, viz. the ambulatory and inpatient sectors. Health care refers here to publicly provided care or private care provided and regulated within the context of a taxfunded or social health insurance system. Administrative expenditure is incurred by government and/or social security-affiliated institutions while running the health system. The grey-filled areas refer to the cost-containment methods that use monetary incentives. The arrows indicate which variables are influenced by the cost-containment method. The straight and dotted arrows refer to a direct and indirect impact on the variables, respectively.

A first way to control the level and growth of health expenditure is via provider payment methods. Providers can be general practitioners, specialists, hospital managers etc. These methods influence the price of health care in a direct way, and may impact upon the quantity of care supplied by providers in an indirect way. Indeed, providers may react to the incentives imbedded in the provider payment method selected, and change the pattern and volume of care. In the first circle at the top of the figure, we mention fee-for-services (FFS), case payment, salaries for providers and per diem payment (although the latter is almost used exclusively for inpatient care). In the second circle at the top of the figure, the capitation payment method is mentioned. It fixes the amount that providers can spend per person entitled to the ambulatory and inpatient services. The third circle at the top of the figure refers to the sectoral budget, whereby health spending by sector is capped. In the second circle at the bottom of the figure, we refer to budget caps on administrative costs. The circle to the right at the bottom of the figure refers to the global budget that caps overall health spending.

Secondly, there are cost-containment instruments that intend to influence the behaviour of patients via patient or user charges, including co-insurance and co-payments. These instruments are intended to impact upon the quantity and type of initial contacts with the health system that patients make in case of need. To a certain extent, they may also influence the volume of referral health care. Reference pricing systems for drugs are also used to encourage patients to substitute to lower priced drugs. Both reference pricing and patient charges are referred to in the circle at the left towards the middle of the figure.

For completeness' sake, other demand and supply-side cost-containment measures are referred to in the white circle towards the middle of the page. These may influence the quantities of care. They may also affect the relative size of patient numbers in the different health sectors. For instance, gatekeeping regulation may impact upon the number of inpatients. As announced earlier, these other 


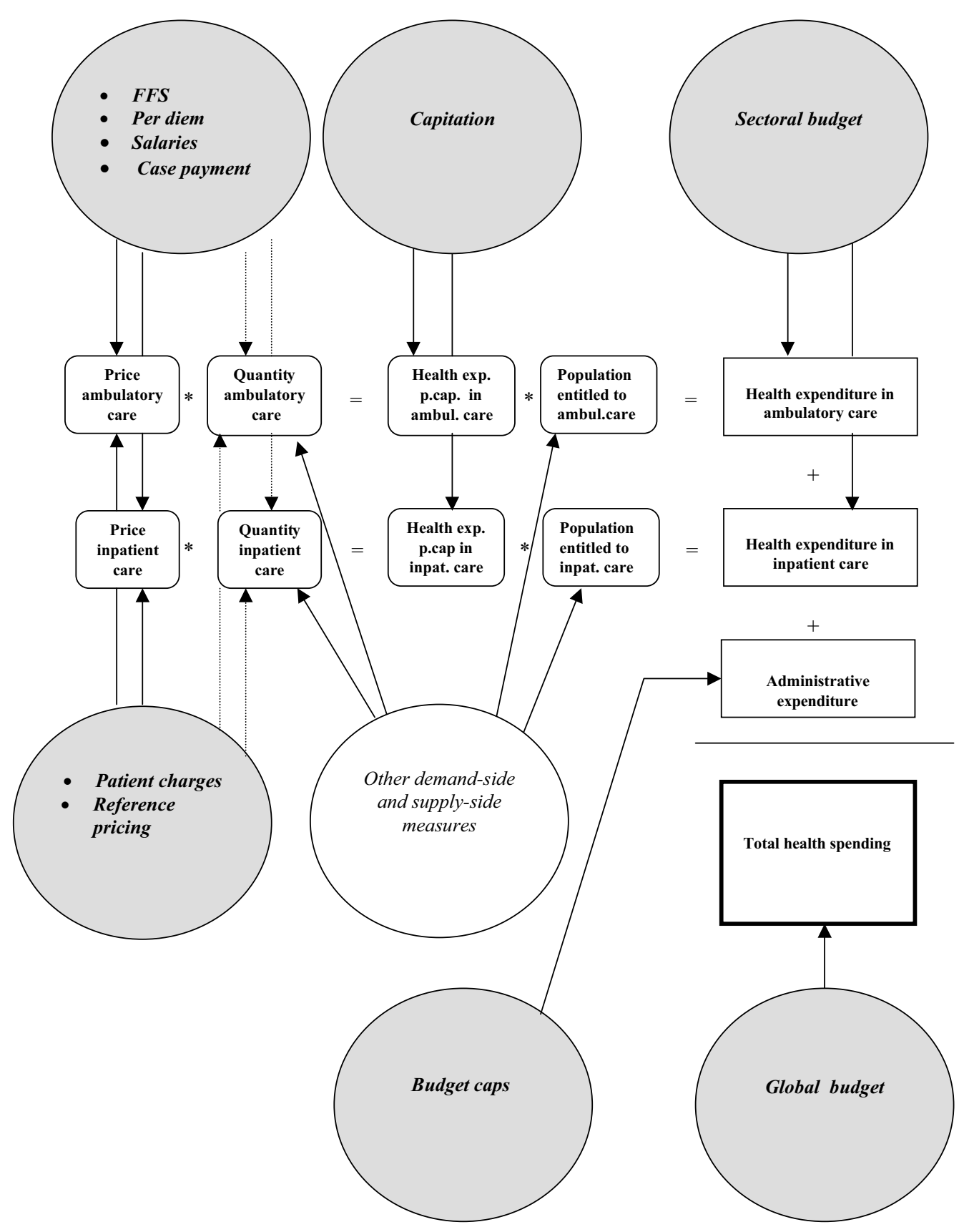

Figure I

Overview of cost-containment methods 
cost-containment measures will not be dealt with in this technical paper.

The overview of cost-containment methods and their impacts does not tell us, however, how successful these are. In addition, cost-containment objectives may not be totally realized, as a result of behaviour of providers or patients that counteract the intended effects. The purpose of the next section is precisely to provide a greater insight into the potential of cost-containment methods intended to influence both provider and patient behaviour. In particular, we review of the empirical literature concerning the use of such methods in high-income countries. Administrative costs will be addressed later.

\section{Policy tools with monetary incentives for cost- containment: a brief overview of experience in high- income countries \\ Provider payment mechanisms: monetary incentives directed at providers}

Fee-for-service (FFS) schedules for both ambulatory care services and inpatient care are used in many systems, especially the social health insurance-based schemes that reimburse patients for costs incurred. Also in mixed systems like the U.S., the government regulated Medicare system for the elderly and disabled uses a resource based relative value scale [2] (RBRVS) for the payment of physician services. It has in common with other fee schedules that it takes account of criteria like time input and skill needed for producing the various health services.

One can expect that application of fee schedules entails important administrative costs, in view of the resources needed for billing, reimbursement of fees, monitoring and possible adjustment of the schedule. There is also the fear that fees create incentives to systematically produce more health services than necessary. One example is from Copenhagen (Denmark) where, in 1987, payment of General practitioners (GPs) changed from a fully capitated payment to a payment that was partly based on fees; the provision of those services that generated extra fees increased significantly [3]. An earlier review on physicians' financial incentives in the U.S. also confirms that production of health services is systematically higher when fees are used to pay providers, as compared to providers that face incentives aiming at controlling treatment and resource use [2].

Another study on differences in health services among 349 physicians found that, after adjusting for patient mix, practices with FFS as the provider payment method had $41 \%$ more hospitalisations than health maintenance organisations using prepayment [4]. We also refer to a study on the determinants of health expenditure in OECD countries. It was found that in countries with FFS payment for physicians, the ratio of physicians per population was positively related to health expenditure [5]. One interpretation is that the possible reduction in average physician income resulting from a greater availability of physicians, seems to be offset via increased health services, at least in a fee-for-service environment.

In a number of circumstances, however, the initial effect of fees on resource use and expenditure can be counteracted. The Japanese social health insurance scheme uses a national fee schedule as one of its key cost-containment instruments. Nearly all procedures and services are subject to this schedule which is applied on a nation-wide basis. Since 1981, the regular revision of the fee schedule has led to relatively low fee levels. In 1995, fees were about one quarter of the Medicare RBRVS fee schedule [6]. It has also led to a relatively modest ratio of health care costs to gross domestic product, namely $7.4 \%$ in 1997 [7]. It is important to note that the overall level of fees in the Japanese system is in fact linked to a kind of overall global budget which is negotiated between the Ministry of Finance and the Ministry of Health and Welfare. With the global budget being capped, selective fee reductions have become logical [8]. The system has also allowed for 'bundling' of fees, with fees becoming flat when a predetermined quantity of services is exceeded. The latter is applied in the case of laboratory tests, for example.

In other social health insurance schemes, explicit budget caps for providers have also been established. Some social health insurance schemes have used this cost-containment method. When Germany used the budget ceiling approach, for example, excessive increases in physicians' services did lead to a proportionate reduction in the level of fees [9]. In Belgium, targets are also set for health insurance expenditure; in 1996 the health insurance budget was overrun and Government reduced the fees by 3 per cent [10]. In the Canadian universal Medicare system, fee schedules are also combined with provincial expenditure caps. In the past across-the-board percentage reduction of fees were observed when these caps were exceeded [11]. In such an environment, doctors are tempted to engage in extra-billing. This has prompted the Canada Health Act of 1984 to stipulate that federal government transfers for health to provinces would be reduced, in the event a province would allow doctors to bill extra charges [12].

Daily payment or per diem payment of hospital services has been used frequently in the past, when funding was still largely open-ended and retrospective. It has been traditionally associated with weak capacity for cost-containment, as it creates incentives to expand length of stay and/ or increase the number of admissions. In order to avoid the effects of such incentives, new ways of prospective payment were designed, among which the case payment. 
Still, a number of countries including Belgium [13] continue to use per diem payments but subject to budget limits. Also in Japan, the biennial fee schedule revisions allow for strict control of hospitalization fees. For instance, in order to stimulate hospitals to shorten average length-ofstay, basic hospitalization fees have been progressively reduced, the per diem rate after ninety days being less than half that of the first fourteen days [37].

Case payment can be used both in ambulatory and inpatient care sector. An example in the former sector is a flat payment per consultation, whatever its nature. Admittedly, there is a similarity with the FFS system. However, in the latter system, the fee schedule is usually more complex and fees vary substantially according to criteria such as length of the consultation, purpose etc. In inpatient care, the best example is the Diagnosis Related Group (DRG) payment method, with hospitals being paid an inclusive flat sum for a patient's treatment according to her diagnostic group. DRGs are used in the USA's Medicare programme for the old aged. DRGs are also used in setting part or the whole of the hospital budget in Austria, Ireland, Finland, Portugal, Spain and Sweden [9].

Case payments such as DRGs are expected to be a good cost-containment device, as hospitals need to provide services within the overall constraint of the flat payment schedule. Evidence on the potential for cost-containment of DRGs is mixed, however. DRGs seem to have resulted in an important decrease in the growth rate of the cost of the U.S. Medicare programme [14]. In Germany, adoption of DRGs in 1993 as an alternative to per diem payment led to both a decrease in the length of stay and a rise in the hospital admissions, resulting in a rise in the number of hospital days per 1000 population. As a result, the rate of increase of hospital costs hardly changed [15].

DRGs may also impact in an indirect way upon the nonhospital sectors. In fact, the net effect of DRGs on total health care costs may well be small, as cost-shifting may take place between the different sectors. In the US, the DRG payment system appeared to have stimulated hospitals to shift patients and costs to outpatient care and longterm care facilities $[16,17]$. Finally, it should be stated that, especially for European countries and Australia, DRGs have been used less as a cost-containment device than as a method to establish case-mix adjusted global hospital budgets.

Capitation payment is a mechanism whereby providers, whether they are GPs or hospitals, receive a flat payment per individual that is covered by a National Health Service system, a social health insurance scheme, or by private health insurance. In return for this flat payment, patients are entitled to services of a pre-defined benefit package.
Capitation payments in outpatient care are used in quite a number of European countries (including Austria, Denmark, Finland, Hungary, Ireland, Italy, the Netherlands and the UK). Capitation payments are not necessarily uniform and can be adjusted for health risks, including old age. In addition, they may be combined with FFS for special services such as in Denmark, Italy and the UK. In the US, capitation payments are pervasive in both outpatient and inpatient care, especially within the framework of Health Maintenance Organizations (HMOs) or managed care plans.

The major characteristic of this payment method is that there is no direct link between the capitation payment and the cost of the health services consumed by an individual patient; in other words, providers are not paid according to the quantity and mix of health services given to that individual. Hence, the incentive to provide excessive health services that other payment methods (such as the FFS and the case payment method) might have, disappears. Instead, there is clearly the incentive to provide less costly treatment. In Finland, where capitation is used in about half of the primary health care centres, outpatient contacts per person are among the lowest in the European Union. The latter phenomenon may be due in part to the capitation system [18]. In the U.S. one study [19] found that capitation for comprehensive health services had led to $40 \%$ fewer inpatient admissions in comparison with FFS systems. In addition, length of stay fell from 5.9 days in 1985 to 3.75 days in 1993. And in the study on health expenditure in OECD countries, referred to earlier, countries that use capitation to reimburse their physicians have lower health spending [5].

When capitation is linked to one category of care only, such as outpatient care, there may be an incentive, however, to underprovide or to refer unnecessarily to higher echelons in the health system. In Hungary, for example, it was found that the referral rate of family physicians that received capitated payments was much higher than in the case of salaried physicians. It is then uncertain what the net impact of capitation on total health care costs will be. In the US, there is debate on whether managed care plans with capitation have been more efficient in controlling costs than other payment methods. Some studies found that HMOs reduced hospital expenditures [20]. Critics [21] have retorted that one should look beyond hospital expenditure though. Their argument is that managed care plans have been known to engage in cost-shifting and in raising administrative costs, so that HMOs do not necessarily reduce or contain total health care spending.

Salaries for doctors are used as a payment method in several OECD countries, including Greece (National Health Service doctors), Spain (60\% of doctors have an income 
that is based on a salary plus a capitation amount), Portugal, Finland and Sweden [9]. Several advantages are advanced: (i) there is no longer an economic incentive to over-provide services, certainly compared to FFS payment; (ii) salaried doctors have an incentive to engage in group consultation, especially in the event of complex cases; (iii) financial planning of health care services becomes easier and administrative costs become lower, as compared to other payment methods, especially FFS.

Disadvantages of a salary system in the public sector are also noted: (i) a salary system reduces incentives for doctors to work productively; (ii) it may lead to low morale for those who work hard but feel they are not rewarded for the extra efforts; (iii) it may then incite the latter to take up work in the private sector, or leave the public sector altogether. Related to the salary payment system as a costcontainment tool, it was found that salary payments are generally associated with lower levels of care [22]. The latter is not unexpected, when comparing the salary method to capitation. Indeed, in order for doctors paid under capitation to increase income, they need to attract more patients. They could do so by offering more services (such as tests or drug prescriptions) speculating thereby that patients would perceive this as high quality care. It is also expected for salaried doctors to offer fewer services than when they would be paid via FFS. In fact, they may even want to minimize their efforts by minimizing the number of patients they see.

Budgets for public health expenditure have been used since a long time in countries with national health systems, such as the UK, Ireland and Denmark. Most levels of the health system in these countries are subject to such budgets. In Denmark, an overall global health budget is negotiated annually and fixed by central and local governments. These budgets are also fixed or 'hard' so that overruns are as good as impossible. Systems that use such budgets are often referred to as closed-end systems as opposed to open-ended financing systems [23].

In other European countries, budgets became increasingly popular since the mid-1980s, as a response to large cost increases in the health sector. Two forms of budget setting are seen: global budgets that, in principle, cover the totality of health care costs and sectoral budgets that are set for specific sectors of the health system. Three further remarks are in order. First, many budgets are rather established as 'targets' and can be exceeded if needed. That is why they are often referred to as 'soft'. In fact, global budgets proved be mostly soft rather than hard. For instance, in 1994 the Government of Belgium established a limit on the real growth of public health expenditure of 1.5 per cent; the limit was respected in 1994 and 1995, but in the two following years, the real growth of the public health expend- iture budget exceeded the limit that was set earlier. Secondly, one has seen a mix of hard and soft sectoral budgets at country level. For example, in France, there are fixed hospital budgets for hospitals whereas there are target budgets for pharmaceuticals, clinical biology, nursing services and office-based doctors. Thirdly, it needs to be verified which costs would not be included in the global budget. In Belgium, for example, administrative costs are excluded from the annual global budgets for health care.

One understands that when budgets are soft, there is hardly a guarantee for success in cost-containment. But even when budgets happen to be hard, they do not automatically lead to cost reductions or lower health expenditure growth rates. They may also be based on historical costs and therefore incorporate important inefficiencies. In the above mentioned OECD inter-country study, for instance, no evidence was found that budget caps for ambulatory and inpatient care lowered total health expenditure [5]. Apart from cost-inefficiencies, other problems have been noted. In France, for example, hard hospital budgets were thought to discourage the adoption of new technologies and to encourage cream skimming. Also in Germany, cream skimming and waiting lists appeared, which has stimulated the abolition of the sectoral hospital budgets [9].

Still when budgets are hard and therefore enforceable, it is possible to contain the expansion of health care costs. When researchers [24] examined inpatient expenditure trends in France between 1960 and 1990, it was concluded that sectoral budgets had been successful in reducing the volume of hospital care. As a result, overall expenditure growth was slowed down. In Ireland, tighter budgetary allocations for the hospital sector were thought to have contributed to the important reduction $(28 \%)$ in the length of stay in hospitals between 1980 and 1993. In Germany budgetary restrictions in January 1993 included a cap on costs of drugs prescribed by office-based physicians; excessive prescribing would result automatically in clawing back the excess from physicians. The number of prescriptions decreased from 795 million in 1992 to 712 million in 1993, with drug expenditure being 25\% lower than in 1992 [25]. Prescriptions seem to have increased again after 1993, but there is still the claim that this policy measure led to savings of about $10 \%$ of the total budget for pharmaceuticals. It is important to bear in mind, however, that a sectoral budget policy may impact on other sub-sectors of the health system. For instance, there was evidence that office-based physicians increased referrals to specialists and hospitals, subsequent to the drug budget policy. This 'substitution' cost has reduced the amount of 'net' global savings in the health sector that was finally realized [26]. 


\section{Policy tools with monetary incentives directed at patients}

Patient charges or user fees are widely used in health systems with the intention to moderate demand of health care. In social health insurance systems, they usually take the form of co-insurance (a fraction of the cost of a health service that is paid by the insured). For example, co-insurance for GP and specialist care can be as high as 30\%, such as in France and Luxembourg. And in the case of prescription drugs, co-insurance schedules exist, with co-insurance rates that are lower, the greater the therapeutic and social value of the prescription drug. These systems also use co-payments (a specified charge for a specified health service), such as for pharmaceuticals and inpatient care in Austria. In tax-funded health systems, cost-sharing through co-payments is also practised. In Sweden, co-payments exist for outpatient care, including dental care, inpatient care and pharmaceuticals. And in the UK, user fees have been introduced for pharmaceutical, dental and ophthalmic health services, as well as for amenity beds [27] in the hospital sector.

From an equity point of view, the use of patient charges ought to be minimal. Access to care may be hampered, certainly among the low-income households. Patient charges on such households may also impact on health. For instance, it was found in the Rand Health Insurance Experiment in the U.S. that low-income patients that were exempted from charges had notable improvements in visual acuity and critical reductions in blood pressure, when compared with non-exempt patients [28]. Recognizing the threats of a negative impact of patient charges on access and health status, exemptions or reduced patient charges were introduced in most systems. More recently, ceilings for patient charges have been introduced in several countries, including Sweden [29], Germany, Belgium, Finland, Ireland and the Netherlands, in order to protect low-income households.

From a cost-containment point of view, patient charges hardly constitute a panacea. For a broad range of health services, patient charges on health services surely tend to decrease their utilisation, but do so rather modestly. To give a quantitative idea about this relatively low impact, it is not uncommon to find [30] that a one percentage increase in patient charges tends to lead to a reduction in utilisation of merely $0.2 \%$.

This downward pressure on utilisation does not imply, however, that overall expenditure would simply drop. In fact, the opposite may occur. It can be shown that, in the context of rising unit costs of health services, increased patient charges and a weak impact of patient charges on health care consumption, total health spending can rise. Cost-reduction is certainly not automatically achieved via patient charges. Admittedly, some degree of cost-contain- ment is achieved, as final health spending is likely to be less compared to health spending in a situation where patient charges are absent.

One also needs to look beyond the impact on health services that are specifically targeted by a cost-containment policy. Indeed, an additional effect of patient charges will be the tendency of patients to substitute the services that are subject to patient charges for other types of care. For instance, additional patient charges on specialist care may incite patients to demand more GP care than before. In that case, the overall net cost-containment effect may be lower than anticipated. In New Hampshire (US), a policy was introduced to limit reimbursement to three drug prescriptions a month for patients with a major psychiatric illness. The use of psychotropic drugs surely dropped, but at the same time demand for the services of mental health centres and hospitals increased. It was estimated that this policy finally resulted in a cost that was 17 times more than it saved [31,38].

Whatever the degree of cost-containment, it is sure that an increase inpatient charges results in a shifting of health care costs to individual patients, which is a move away from risk-sharing across all population groups.

Reference price systems for pharmaceuticals are a relatively new cost-containment method and has been introduced since the late eighties. Basically, a cluster of similar drugs is associated with one specific price accepted by government for reimbursement purposes. Should a physician prescribe a drug priced above this 'reference' price, it is the patient who will pay the difference. This policy is meant to increase the cost-consciousness of patients and to incite them to demand reference priced drugs. New Zealand was the first to introduce reference pricing, to be followed in Europe by Germany (1989), and then the Netherlands (1991), Denmark and Sweden (1993), and Italy (1996) [9]. Little information is available on patients' behaviour with regard to these systems.

At a macroeconomic level, however, reference price systems do not appear to have been successful. This is because reference price systems apply to interchangeable drugs only. New and innovative drugs are therefore not likely to be covered by these systems, and escape price control. In the Netherlands, Germany and Sweden, growth of the sales of products that were not covered by the reference price system, easily outweighed the cost-savings generated by prescribing reference-priced drugs [9].

\section{Levels and trends in administrative costs}

Administrative costs are defined here as the public part of the costs of health administration and social insurance at all levels of government. Included in these costs are costs 
as a result of planning, management, regulation, and collection of funds and handling of claims of the delivery system [32]. Obviously, these costs can in principle also be targeted for cost-containment. It is important to consider these as well, since excessive administrative costs entail considerable opportunity costs: funds that are spent unwisely can not be spent on health care itself, an extension of the benefit package, an expansion of population coverage etc.

An earlier comparative analysis of administrative costs in a sample of OECD countries showed that in 1990 public administrative expenditures as a percentage of total public expenditure on health varied from $0.2 \%$ to $7.1 \%$ [33]. It was noted in this analysis that insurance-based systems tend to be relatively more expensive than general revenue based systems. More recent data (from 1990 to 1999) for a sample of OECD countries have confirmed this finding, the mean share of administrative costs in health spending being $2.2 \%$ and $4.2 \%$ for general revenue-based an insurance-based systems, respectively. We thus find that 'on average' the insurance-based systems spend proportionally more administrative resources than in the tax-based systems. This divergence is not absolute, however. Note for example that in 1998, Japan devoted a modest $2 \%$ of its health spending to administration of its social health insurance system. The latter result can be explained to a large extent by the presence of one 'Social Insurance Medical Care Fee Payment Fund' [34] where the totality of claims and reimbursements is processed, as opposed to systems whereby different pools or funds are involved in such payments and therefore are each confronted with additional administrative costs.

It has been mentioned that a lack of harmonized data may make comparisons difficult. For example, the data for France appear to be incomplete, as they cover the costs of the government health administration only, and not those of the social security institutions. But even after improving on the data comparability, an important degree of dispersion between the two types of health financing systems is likely to remain. Still, a comparison of the levels of shares administrative cost in total spending is not straightforward because, in principle, one ought to take account of the differences in the 'products' supplied by the administration. Differences in administrative costs are not only due to the amount of transactions such as claims processing in insurance schemes. They may also be due to differences in the variety of information services to patients (e.g. about benefit entitlements, health information), and in the degree to which the administration is involved in monitoring the quality of health services, in efforts to contain spending and in data-processing of clinical and financial information. We recognize that the explanation of differences in administrative costs would merit further attention. This is beyond the scope of the present technical paper, however.

We propose now to look into the trends of administrative expenditure as a share of total public health expenditure. It is interesting to observe that these shares, whether from insurance based or tax based health systems, were subject to a negative yearly trend of $-0.1 \%$ over the last decade. A reduction over time of the shares of administrative costs is not unexpected. In tax-based systems where national and/ or district administrative bodies have the responsibility to finance health care for target population groups, economies of scale in administration are likely to arise. Likewise, in the case of insurance-based schemes, average costs of administration tend to drop with an increasing number of patient claims; in other words, there are usually economies of scale in processing of claims. In the U.S. for example, evidence was reported on the existence of economies of scale in the non-profit Blue Shield health insurance plans. It was also estimated, for the year 1976, that these plans could minimize average administrative cost when processing 17.7 million claims per year [35]. Another reason for a downward trend is the increasingly efficient way of handling administrative tasks as a result of technological advances such as computerization of billing, settling claims and records [33]. In France for example, a smart card or 'carte vitale' was introduced in 1999 [36], which significantly facilitates access to care and reimbursement operations. Improvements in technology and subsequent productivity have also permitted a reduction in administrative labour costs: sickness funds reduced their number of employees by 5 percent between 1980 and 1990. In other countries with an insurance-based system, measures were introduced to limit administrative spending of health insurance funds.

Policy tools with monetary incentives also exist, however. We refer especially to budget caps on administrative resources managed by various administrative units in the health system. In Belgium, for example, capped budgets for administration are established for its seven health insurance funds; a typical budget consists of a fixed part, which is granted unconditionally, and a variable part which depends on performance of the fund. Note that for the year 2002, the estimated share of these funds' administrative costs in total reimbursements is estimated to be $4.63 \%$. Empirical evidence in the other OECD countries about the effects of specific measures introduced to contain administrative costs is not readily available, however.

\section{The potential role of the policy instruments in cost- containment: a summary}

In Table 1, we summarize the potential role of the various instruments intended to contain health care costs, based on the review of the international literature. Additional 
Table I: The potential of monetary incentives in cost-containment policies

\begin{tabular}{|c|c|c|c|c|}
\hline $\begin{array}{l}\text { Cost-containment } \\
\text { instrument }\end{array}$ & Incentive directed at & Relevant category of care & $\begin{array}{l}\text { Potential for cost- } \\
\text { containment }\end{array}$ & Additional comments \\
\hline Fee-for-service & Providers & Outpatient and inpatient care & Low & $\begin{array}{l}\text { - The upward effect of fees on costs can be neutralized, } \\
\text { when hard budgets are introduced }- \text { The structure and } \\
\text { level of fees in the FFS schedule can be changed for cost- } \\
\text { containment purposes. }\end{array}$ \\
\hline Per diem payment & Providers & Inpatient care & Low & $\begin{array}{l}\text { The upward effect of per diem payments on hospital } \\
\text { costs can be neutralized, when hard hospital budgets are } \\
\text { introduced }\end{array}$ \\
\hline Case payment & Providers & Inpatient and outpatient care & Medium & $\begin{array}{l}\text { The net effect on cost-containment may be reduced by } \\
\text { cost-shifting to other health sectors }\end{array}$ \\
\hline Capitation & Providers & Inpatient and outpatient care & Medium & $\begin{array}{l}\text { The net effect on cost-containment may be reduced by } \\
\text { cost-shifting to other health sectors }\end{array}$ \\
\hline Salaries & Providers & $\begin{array}{l}\text { Inpatient and outpatient care } \\
\text { (worktime of providers only) }\end{array}$ & High & $\begin{array}{l}\text { The overall on cost-containment of national health } \\
\text { expenditure depends on provider payment mechanisms } \\
\text { introduced for other types of care }\end{array}$ \\
\hline Budgets & Providers & Inpatient and outpatient care & High & $\begin{array}{l}\text { - The cost-containment efficiency of budgets is low, when } \\
\text { budgets are 'soft' - Cost-shifting between sectors may } \\
\text { reduce the overall cost-containment potential }\end{array}$ \\
\hline Patient charges & Patients & Inpatient and outpatient care & Low & $\begin{array}{l}\text { Cost-reductions may be seen with important increases in } \\
\text { patient charges, combined with the existence of medium } \\
\text { to high price sensitivity }\end{array}$ \\
\hline Reference price system & Patients & Pharmaceuticals & Medium & $\begin{array}{l}\text { Cost-containment potential will be low, when prescribing } \\
\text { of drugs outside the reference-price system remains } \\
\text { important }\end{array}$ \\
\hline
\end{tabular}

comments are provided in the Table as well, referring to possible pitfalls or circumstances where the expected impact on cost-containment can be reduced or neutralized. Methods to contain administrative costs are not summarized in Table 1, because of weak empirical evidence on the effects of specific cost-containment methods. Budget caps on administrative costs along with the application of technological advances are likely to be good instruments for cost-containment. However, more international evidence needs to be generated before arriving at generally applicable lessons.

Related to measures that impact upon the supply of health services, we offer the following conclusions. One first message from Table 1 is that salaries and budgets do in principle have a high cost-containment potential. There are two caveats, however. One is that while salaries may affect an important fraction of health care costs, there is no certainty that costs of other inputs are contained. Secondly, budgets may be of the soft kind. Or, budgets are being used but permit sizeable growth of health care costs. A second message is that case payment and capitation do have some cost-containment potential. There is an important risk of shifting of costs to other sub-sectors, however. Thirdly, fee-for-services and per diem payments usually do not have a cost-containment potential. They can even stimulate costs. An important caveat, however, is that a potentially negative effect on cost-containment can be counteracted if hard budgets are put in place.

Concerning measures directed at the demand of health services, we submit, first, that patient charges do not appear to be a successful cost-containment tool, as patients' sensitivity to such charges is usually quite modest. Apart from the expected modest impact on cost-containment, there is also the adverse impact of patient charges on equity in access, which is of special concern to low-income households. Secondly, a reference price system may also contribute to cost-containment, but the degree of success depends on whether prescribing of drugs outside the reference price system remains important.

\section{Competing interests}

No competing interests.

\section{Authors' contributions}

Guy Carrin: analysis of evidence and interpretation, writing of the manuscript

Piya Hanvoravongchai: references, data interpretation and revision of manuscript

\section{Acknowledgments}

The authors are grateful to the members of the Health Financing Group in the Department of Health Financing and Stewardship (WHO, Geneva) and to two referees for useful suggestions and comments. Also thanks to Manique Abayasekara for assistance with the final layout of the manuscript. All remaining errors, however, are those of the authors. In addition, all views expressed in this paper are entirely those of the authors and do not necessarily represent those of the WHO.

\section{References}

I. Gray G: Access to medical care under strain: new pressures in Canada and Australia Journal of Health Politics, Policy and Law 1998, 23(6): $905-947$.

2. Feldstein P: Health Care Economics Albany: Delmar Publishers 1999. 
3. Rice T: The Economics of Health Reconsidered Chicago: Health Administration Press 1998.

4. Greenfield $S$ et al: Variations in resource utilization among medical specialties and systems of care JAMA 1992, 267(I 2): 1624-1630.

5. Gerdtham UG and Jonsson B: International comparisons of health expenditure: theory, data and econometric analysis In Handbook of health economics Volume IA. Edited by: Culyer AJ, Newhouse JP. Amsterdam: Elsevier; 2000:II-53.

6. Ikegami $\mathrm{N}$ and Campbell JC: Medical care in Japan The New England Journal of Medicine 1995, 333(19): I 295-1299.

7. World Health Organization: The World Health Report 200 Geneva 200I.

8. Ikegami $\mathrm{N}$ and Campbell JC: The art of balance in health policy: maintaining Japan's low-cost, egalitarian system New York: Cambridge University Press 1998.

9. Mossialos E and Le Grand J: Cost Containment in the EU: an overview In Health care and cost containment in the European Union Edited by: Mossialos E, Le Grand J. Aldershot: Ashgate Publishing Ltd; 1999: I-I54.

10. Crainich D and Closon MC: Cost containment and health care reform in Belgium In Health care and cost containment in the European Union Edited by: Mossialos E, Le Grand J. Aldershot: Ashgate Publishing Ltd; 1999:219-266.

II. Barer ML, Lomas J and Sanmartin C: Re-minding our Ps and Qs: Medical cost controls in Canada Health Affairs 1996, I5(2):216-234.

12. Canada's Health Care System [http://www.hc-sc.gc.ca/]

13. European Observatory on Health Care Systems Health Care Systems in Transition: Belgium Copenhagen 2000.

14. Coulam RF and Gaumer GL: Medicare's prospective payment system: a critical appraisal Health Care Financing Review, Annual Suppl:45-77.

15. Schulenbury JM: Forming and reforming the market for thirdparty purchasing of health care: Social Science and Medicine 1994, 39(10):|473-8|.

16. Miller $M$ et al.: Service mix in the hospital outpatient department: implications for Medicare payment reform Health Services Research 1995, 30(I):59-77.

17. Carroll NV and Erwin WG: Patient shifting as a response to Medicare prospective payment Medical Care 1987, 25: I I6I-7.

18. Järvelin J: Health care systems in transition Finland: WHO, Copenhagen 2002.

19. Tabbush $V$ and Swanson G: Changing paradigms in medical payment Archives of Internal Medicine 1996, 156:357-60.

20. Miller R and Luft H: Managed Care Performance since 1980: A literature analysis Journal of the American Medical Association 1994:1512-1519.

21. Sullivan K: On the 'efficiency' of managed care plans Health Affairs 2000:139-148.

22. Gosden $T$, Pedersen $L$ and Torgerson D: How should we pay doctors? A systematic review of salary payments and their effect on doctor behaviour QJ Med 1999, 92:47-55.

23. Jegers M, Kesteloot K, De Graeve D and Gilles W: A typology for provider payment systems in health care Health Policy 2002, 60(3):255-273.

24. Redmon $P$ and Yakoboski $P$ : The nominal and real effects of hospital budgets in France Inquiry 1995, 32: 174- 183.

25. Bloor $\mathrm{K}$ and Freemantle $\mathrm{N}$ : Lessons from international experience in controlling pharmaceutical expenditure II: influencing doctors British Medical Journal 1996, 3 I 2: I525-I527.

26. Schöffski O, Schulenberg JM and Graf von der: Unintended effects of a cost-containment policy: results of a natural experiment in Germany Social Science and Medicine 1997, 45(10): 1537-1539.

27. European Observatory on Health Care Systems: Health Care Systems in Transition United Kingdom. WHO Copenhagen .

28. Brook H, Ware JE and Rogers WE et al: Does free care improve adults' health? New England Journal of Medicine 1983, 309(23): 1 1426-34.

29. Andersen R, Smedby B and Vagero D: Cost containment, solidarity and cautious experimentation: Swedish dilemmas Social Science and Medicine 200I, 52:I 195-1204.

30. Zweifel P and Manning WG: Moral hazard and consumer incentives in health care In Handbook of Health Economics Volume IA. Edited by: Culyer AJ, Newhouse JP. Amsterdam: North Holland; 2000:409-459.
31. Soumerai SB, McLaughlin TJ, Ross-Degnan D, Casteris CS and Bollini $P$ : Effects of limiting Medicaid drug-reimbursement benefits on the use of psychotropic agents and acute mental health services by patients with schizophrenia New England Journal of Medicine 1994, 331:650-655.

32. OECD: OECD Health Data 200I Comparative Analysis of $\mathbf{3 0}$ Countries Paris: OECD 200I.

33. Poullier JP: Administrative costs in selected industrialized countries Health Care Financing Review 1992, 13(4): 167-172.

34. Ministry of Health and Welfare, Japan: Outline of Social Insurance in Japan Tokyo 1999.

35. Kass DI and Pautler PA: The administrative costs of non-profit health insurers Economic Inquiry 198I, 29(3):5I5-52I.

36. Duriez M: Le système de santé en France Paris: Haut Comité de Santé Publique 2000

37. Ikegami $\mathrm{N}$ and Campbell JC: Health care reform in Japan: The virtues of muddling through Health Affairs 1999, 18(3):56-75.

38. Freemantle $\mathrm{N}$ and Bloor $\mathrm{K}$ : Lessons from international experience in controlling pharmaceutical expenditure. I: influencing patients British Medical Journal I 996, 3 | 2: | 469- | 47 |.
Publish with Biomed Central and every scientist can read your work free of charge

"BioMed Central will be the most significant development for disseminating the results of biomedical research in our lifetime. "

Sir Paul Nurse, Cancer Research UK

Your research papers will be:

- available free of charge to the entire biomedical community

- peer reviewed and published immediately upon acceptance

- cited in PubMed and archived on PubMed Central

- yours - you keep the copyright
BioMedcentral 